\title{
Comparison of fresh and frozen thawed embryo transfer in terms of clinical pregnancy rate
}

\author{
Adaboina Anitha*, Burri Sandhya Rani
}

Department of Obstetrics and Gynecology, Laxmi Narasimha Hospital, Hanamkonda, Warangal, Telangana, India

Received: 19 November 2019

Revised: 04 December 2019

Accepted: 30 December 2019

\section{*Correspondence:}

Dr. Adaboina Anitha,

E-mail: anithaadaboina@gmail.com

Copyright: (c) the author(s), publisher and licensee Medip Academy. This is an open-access article distributed under the terms of the Creative Commons Attribution Non-Commercial License, which permits unrestricted non-commercial use, distribution, and reproduction in any medium, provided the original work is properly cited.

\section{ABSTRACT}

Background: In a standard IVF (in-vivo fertilization) procedure, the embryos formed after the fertilization of male and female gametes are allowed to grow for 3-5 days and then transferred back to the uterine cavity of the female, where they might get attached and start to grow. Objective of this study was to compare clinical pregnancy rate of fresh embryo transfers and frozen-thawed embryo transfers.

Methods: This is a retrospective case control study in patients undergoing IVF /ICSI cycles from January 2018 to December 2018 were enrolled in assisted reproduction. Total of 200 women which contains 118 fresh embryo transfers and 82 frozen-thawed embryo transfers are studied.

Results: Clinical pregnancy rates of fresh cleavage-stage embryo transfers compared with frozen-thawed cleavagestage embryo transfers, were (53.3\% versus $39.6 \%)$. Ectopic pregnancy is also significant in comparison. In patients under 35 years of ages and (57.1\% versus $12.5 \%$ ). In patients older than 35 years old, respectively. The multiple pregnancy rates, abortion rates and ectopic pregnancy rates did not differ significantly among the groups. Multiple pregnancy rate and abortion rate is significantly high in frozen-thawed blastocyst transfer than fresh embryo transfer. Whereas the ectopic pregnancy rates had no difference in both groups.

Conclusions: The clinical pregnancy rates in fresh embryo transfer is high than that of frozen-thawed blastocyst.

Keywords: Ectopic pregnancy, Fresh embryo transfer, Frozen-thawed blastocyst, In-vivo fertilization, Multiple pregnancy

\section{INTRODUCTION}

In a standard IVF (in-vivo fertilization) procedure, the embryos formed after the fertilization of male and female gametes are allowed to grow for 3-5 days and then transferred back to the uterine cavity of the female, where they might get attached and start to grow. This is known as fresh IVF cycle or IVF with fresh embryo transfer. In a typical IVF cycle, especially for a young couple, multiple embryos are formed. Some may even have 8-10 embryos and if they are considered to be good quality, the doctor may decide to freeze them at that stage. Most doctors will transfer only one or two embryos in a fresh cycle and if none of them bring a positive result, you can rely on the frozen embryos for another chance, without having to repeat the whole IVF process. One or two of your frozen embryos will be thawed (de-frozen) and transferred back to your uterine cavity. This process is called frozen embryo transfer (FET).

On the other hand, embryo cryopreservation has also provided additional clinical safety in the presence of ovarian hyperstimulation. ${ }^{1}$ However, the pregnancy rate in frozen-thawed embryo transfer cycles is usually lower 
than that of fresh transferred embryos (In addition, the chance of live birth following FET is further reduced by the increased incidences of pregnancy damage). ${ }^{2}$ More concrete research is required in this direction before we can confidently adopt the freeze-all policy for embryos in IVF/ICSI cycles, even though many doctors across the world are following this practice. Recently, many researches showed frozen-thawed embryo transfers can enhanced the embryo utilization rate and improve the success rate. Estrogen-progesterone (E-P) cycles or nature cycles were adopted in frozen-thawed embryo transfer. ${ }^{3}$ This study was aimed to compare clinical pregnancy rate of fresh embryo transfers and frozenthawed embryo transfers.

\section{METHODS}

The study performed from January 2018 to December 2018 in assisted reproduction in total of 200 women which contains 118 fresh embryo transfers and 82 frozenthawed embryo transfers.

\section{Inclusion criteria}

- Polycystic ovarian syndrome

- Unexpected infertility

- Endometriosis

- Male factor infertility

- Bilateral tubal block

- Pelvic inflammatory disease

- Uterine fibroids

- Hypothalamic Amenorrhea.

\section{Exclusion criteria}

- Couple with family history of genetic disease

- Embryo or Oocyte donation

- Cancer patients requiring surgery, chemotherapy or radiotherapy

- Gestational surrogacy.

All techniques of IVF including ovarian stimulation, harvesting of oocytes, insemination with specially prepared sperm and embryo culture in the laboratory will be according to local protocols. Once the embryos have been cultured up to day 2 or 3 , women with 3 or more suitable quality embryos will be randomized to either fresh embryo transfer (control arm) or FET (Intervention arm).

Control arm women allocated to the control arm will either undergo embryo transfer at cleavage stage or extended culture and transfer at blastocyst stage according to local policy. A maximum of 2 embryos or blastocysts will be replaced according to the standard protocol under transabdominal ultrasound guidance. Luteal phase support is given according to local protocols.
Intervention arm for those allocated to the intervention arm fresh embryo transfer will not be undertaken. Embryos will be frozen by vitrification or slow freezing at cleavage or blastocyst stage according to standard agreed local protocols. Women will be contacted after 4 weeks and arrangements made for frozen embryo transfer.

After at least 4 weeks women will attend the IVF clinic for the transfer of thawed frozen embryos, either in a natural cycle (in ovulatory women who have regular menstrual cycles) or in a hormonally supported cycle using physiological doses of estrogen and progestogens to mimic normal cyclical changes within the endometrium. The choice between replacing frozenthawed embryos in a natural or hormonally mediated cycle will be a decision driven by the clinical circumstances.

Embryos or blastocysts will be thawed according to local protocols. The transfer is performed by the team clinician with a maximum of 2 embryos or blastocysts being replaced according to the standard protocol under transabdominal ultrasound guidance. Luteal phase support is given at the discretion of the physician.

Embryo cryopreservation techniques and transfer protocols

Morphological evaluation was done on the second day after oocyte recapture. Number of blastomeres and cytoplasmic fragmentation was assessed. Two embryos with good or excellent quality were transferred in fresh cycles. All the extra embryos with less than $30 \%$ fragmentation were cryopreserved by vitrification method.

During two step loading protocols, embryos were loaded with equilibration solution containing $7.5 \%$ dimethyl sulfoxide (DMSO) and $7.5 \%$ ethylene glycol (EG) in Ham's F-10 media supplemented with $20 \%$ patient serum for $5-15 \mathrm{~min}$ at room temperature. Once the primary shrinkage and recovery, embryos were aspirated and placed into the vitrification solution $15 \% \mathrm{EG}, 15 \%$ DMSO and $0.5 \mathrm{M}$ sucrose in Ham's F-10 medium supplemented with $20 \%$ patient serum for $50-60 \mathrm{sec}$ at room temperature. The embryos then were loaded by a thin glass capillary tube into the cryotop and the samples were immediately submerged into liquid nitrogen for at least 2 months.

For thawing, cryoprotectants were removed step by step using embryo thawing media by insertion the Cryotop in thawing solution (1 M sucrose) for 50-60 sec and next into dilution solution ( $0.5 \mathrm{M}$ sucrose) for $3 \mathrm{~min}$, after that embryos placed in another dilution solution of $0.25 \mathrm{M}$ sucrose for $3 \mathrm{~min}$, all at room temperature. The thawed embryos were placed 4-5 times into washing solution (Ham's F-10+20\% serum) before incubation. After embryo transferred to culture medium, they considered 
morphologically survived by $50 \%$ or more intact blastomeres and no injury to zonapllucida. Only intact or partly damaged embryos were transferred.

Oral estradiol Valerat at the dose of $6 \mathrm{mg}$ per day from the second day of menstrual cycle for Endometrial preparation was until the endometrial thickness reached greater than $8 \mathrm{~mm}$, and then $100 \mathrm{mg}$ progesterone in oil was injected or Cyclogest $400 \mathrm{mg}$ was used daily. Estradiol and progesterone administered continuously until observation of fetal heart activity by ultrasound. Embryo transfer was done 3 days after the beginning of progesterone injection.

\section{Follow up strategy}

A pregnancy test will be carried out 2 weeks after embryo transfer in both arms. All women who have a positive pregnancy test 2 weeks after embryo transfer will undergo a transvaginal ultrasound scan to identify the presence and number of a gestation sac with a fetal heart signifying an ongoing pregnancy. Pelvic scan will be repeated at 8 weeks, 12 weeks, 24 weeks and 36 gestation for fetal growth.

\section{Statistical analysis}

All statistical calculations were done by SPSS software. Logistic regression analysis was used to adjust the confounding factors to compare the embryo clinical pregnancy rates between the two groups. $\mathrm{P}<0.05$ was considered statistically significant.

\section{RESULTS}

A total of 200 women which contains 118 fresh embryo transfers and 82 frozen-thawed embryo transfers.

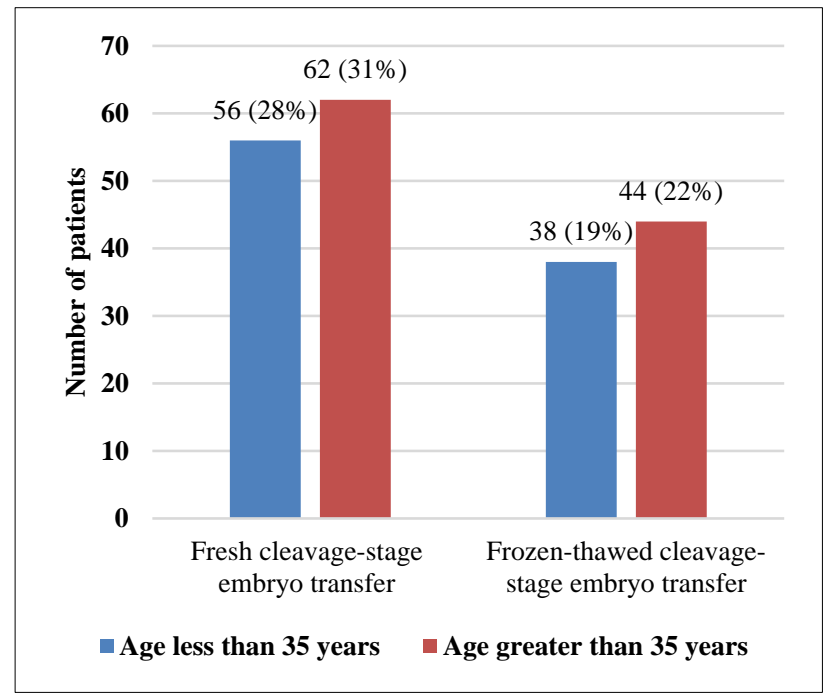

Figure 1: Age distribution in present study.

Table 1: Demographic details of patients studied.

\begin{tabular}{|c|c|c|c|}
\hline Variable & $\begin{array}{l}\text { Fresh cleavage-stage } \\
\text { embryo transfer }(n=118)\end{array}$ & $\begin{array}{l}\text { Frozen-thawed cleavage-stage } \\
\text { embryo transfer }(n=82)\end{array}$ & p-value \\
\hline & Mean \pm SD & Mean \pm SD & $>0.05(\mathrm{NS})$ \\
\hline Body mass index & $19.23 \pm 3.43$ & $19.45 \pm 3.56$ & $>0.05(\mathrm{NS})$ \\
\hline Male age (year) & $40.4 \pm 5.33$ & $40.1 \pm 5.67$ & $>0.05(\mathrm{NS})$ \\
\hline Infertility duration (year) & $8.45 \pm 4.56$ & $8.78 \pm 4.87$ & $>0.05(\mathrm{NS})$ \\
\hline Basal FSH (IU/ml) & $6.7 \pm 2.98$ & $6.6 \pm 3.12$ & $>0.05(\mathrm{NS})$ \\
\hline Embryos transferred & $2.87 \pm 0.81$ & $2.67 \pm 0.83$ & $>0.05(\mathrm{NS})$ \\
\hline
\end{tabular}

Table 2: The frequency distribution of pregnancy rate based on etiology of infertility.

\begin{tabular}{|lllll|}
\hline \multirow{2}{*}{ Etiology of infertility } & \multicolumn{2}{l}{$\begin{array}{l}\text { Fresh cleavage-stage } \\
\text { embryo transfer }(\mathbf{n}=\mathbf{1 1 8})\end{array}$} & $\begin{array}{l}\text { Frozen-thawed cleavage-stage } \\
\text { embryo transfer }(\mathbf{n}=\mathbf{8 2})\end{array}$ \\
\hline & Number of patients & Percentage $\mathbf{( \% )}$ & Number of patients & Percentage $(\mathbf{\%})$ \\
\hline Male factor & 32 & 16 & 23 & 11.5 \\
\hline Poly cystic ovary & 58 & 29 & 42 & 21 \\
\hline Tubal factor & 4 & 2 & 3 & 1.5 \\
\hline Endometriosis & 2 & 1 & 2 & 1 \\
\hline Hypothalamic amenorrhea & 1 & 0.5 & 1 & 2 \\
\hline Unexplained & 11 & 5.5 & 4 & 3.5 \\
\hline Mixed & 10 & 5 & 7 & $\mathbf{4 1}$ \\
\hline Total & $\mathbf{1 1 8}$ & $\mathbf{5 9}$ & $\mathbf{8 2}$ & \\
\hline
\end{tabular}

Out of 200 women in study 94 women are below 35 $(47 \%)$ and $106(53 \%)$ are above 35 years.
All the parameters in the study compared in 2 groups are insignificant so both groups can be compared with. 
Polycystic ovary is most common etiology of infertility in both groups followed by male factor.

For patients under 35 years of age, the numbers of good embryo transferred were different in the fresh cleavagestage embryo transfers and frozen-thawed blastocyst transfers. The clinical pregnancy rates after the fresh embryo transfers and frozen-thawed transfers were different $(53.3 \%$ versus $39.6 \%)$ which is statically significant on comparison. Ectopic pregnancy is also significant in comparison. But other clinical outcomes, for example multiple pregnancy rates, abortion rates had no difference.
For patients older than 35 years old, the clinical characteristics dose of gonadotropin, retrieved of oocyte, and number of good embryos transferred were different significantly between the fresh embryo transfers and frozen-thawed blastocyst transfers clinical pregnancy $(57.1 \%$ versus $12.5 \%)$ is also significantly high in fresh embryo transfer than that of frozen-thawed blastocyst. Multiple pregnancy rate and abortion rate is significantly high in frozen-thawed blastocyst transfer than fresh embryo transfer. Whereas the ectopic pregnancy rates had no difference in both groups.

Table 3: Comparison with fresh cleavage-stage embryo transfers and frozen-thawed blastocyst transfers in women age less than 35 years.

\begin{tabular}{|llll|}
\hline & $\begin{array}{l}\text { Fresh cleavage-stage } \\
\text { embryo transfer }(\mathbf{n = 6 0})\end{array}$ & $\begin{array}{l}\text { Frozen-thawed cleavage-stage } \\
\text { embryo transfer }(\mathbf{n}=\mathbf{5 8})\end{array}$ & p-value \\
\hline Dose of gonadotropin & $29.44 \pm 9.33$ & $29.77 \pm 9.12$ & $>0.05(\mathrm{NS})$ \\
\hline Retrieved of oocyte & $14.43 \pm 6.99$ & $15.23 \pm 7.44$ & $>0.05(\mathrm{NS})$ \\
\hline Endotrium thickness & $10.65 \pm 2.53$ & $9.45 \pm 1.65$ & $>0.05(\mathrm{NS})$ \\
\hline No of embryo transferred & $1.67 \pm 0.32$ & $2.23 \pm 0.51$ & $>0.05(\mathrm{NS})$ \\
\hline No of good embryo transferred & $1.83 \pm 0.21$ & $1.76 \pm 0.51$ & $>0.05(\mathrm{NS})$ \\
\hline Clinical pregnancy rate & $53.3 \%(32 / 60)$ & $39.6 \%(21 / 58)$ & $<0.05(\mathrm{~S}) *$ \\
\hline Multiple pregnancy rate & $31.2 \%(10 / 32)$ & $33.33 \%(12 / 29)$ & $>0.05(\mathrm{NS})$ \\
\hline Abortion rate & $6.6 \%(4 / 60)$ & $6.8 \%(4 / 58)$ & $>0.05(\mathrm{NS})$ \\
\hline Ectopic pregnancy rate & $1.6 \%(1 / 60)$ & 0 & $<0.05(\mathrm{~S}) *$ \\
\hline
\end{tabular}

Table 4: Comparison with fresh cleavage-stage embryo transfers and frozen-thawed blastocyst transfers in women age greater than 35 years.

\begin{tabular}{|c|c|c|c|}
\hline & \multicolumn{2}{|l|}{$\geq 35$ years } & \multirow[b]{2}{*}{ p-value } \\
\hline & $\begin{array}{l}\text { Fresh cleavage-stage } \\
\text { embryo transfer }(n=42)\end{array}$ & $\begin{array}{l}\text { Frozen-thawed cleavage- } \\
\text { stage embryo transfer }(n=40)\end{array}$ & \\
\hline Dose of gonadotropin & $43.93 \pm 15.95$ & $39.69 \pm 15.39$ & $<0.05(\mathrm{~S})^{*}$ \\
\hline Retrieved of oocyte & $10.47 \pm 6.61$ & $11.46 \pm 6.98$ & $<0.05(\mathrm{~S})$ \\
\hline Endotrium thickness & $10.49 \pm 2.40$ & $9.97 \pm 1.74$ & $>0.05(\mathrm{NS})$ \\
\hline No of embryo transferred & $1.90 \pm 0.49$ & $1.99 \pm 0.79$ & $>0.05(\mathrm{NS})$ \\
\hline No of good embryo transferred & $1.93 \pm 0.87$ & $2.28 \pm 0.51$ & $<0.05(\mathrm{~S})$ \\
\hline Clinical pregnancy rate & $57.1 \%(24 / 42)^{*}$ & $12.5 \%(6 / 40)^{*}$ & $<0.05(\mathrm{~S})^{*}$ \\
\hline Multiple pregnancy rate & $25 \%(6 / 24)$ & $16.67 \%(1 / 6)$ & $<0.05(\mathrm{~S})^{*}$ \\
\hline Abortion rate & $9.5 \%(7 / 42)$ & $33.33 \%(2 / 6)$ & $<0.05(\mathrm{~S})^{*}$ \\
\hline Ectopic pregnancy rate & 0 & 0 & $>0.05(\mathrm{NS})$ \\
\hline
\end{tabular}

\section{DISCUSSION}

The present comparative study was carried out to provide a better understanding of the clinical factors in predicting the clinical pregnancy rate of fresh embryo transfers and frozen-thawed embryo transfers. A total of 200 women which contains 118 fresh embryo transfers and 82 frozenthawed embryo transfers have participated in study. Both groups are compared with each other. In present study Polycystic ovary is most common etiology of infertility in both groups followed by male factor. (29\% and 21\%) (Table 1 and 2). In our study under 35 years of age, the numbers of good embryo transferred were different in the fresh cleavage-stage embryo transfers and frozen-thawed blastocyst transfers. In our study 94 women are below 35 $(47 \%)$ and $106(53 \%)$ are above 35 years (Figure 1$)$ 
The clinical pregnancy rates after the fresh embryo transfers and frozen-thawed transfers were different $(53.3 \%$ versus $39.6 \%)$ which is statically significant on comparison. (Table 3) Ectopic pregnancy is also significant in comparison. But other clinical outcomes, for example multiple pregnancy rates, abortion rates had no difference. The influence of blastocyst after cryopreservation may be little. The cleavage-stage embryos did not induce self-selection through the extended culture. So, the ability of resistance may be weaker than blastocyst. The result was in according with many studies. ${ }^{4,5}$ In previous reports, a reduced pregnancy rate following FET, as well as, following the transfer of fresh embryos was recorded with increasing maternal age.

According to the study by Ashrafi et al the women age did not affect pregnancy rate in FET protocol. ${ }^{6}$ They concluded that the quality of embryos was crucial factor determining the success of FET. Other studies also revealed the FSH level caused a remarkable effect on pregnancy rate in FET. $^{7}$ In another investigation, Kassab et al reported inverse correlation between basal serum FSH levels before fresh IVF/ICSI cycle with pregnancy outcome in FET cycles. ${ }^{8}$ The other factors consisting reason for embryo cryopreservation, ovulationstimulating protocol, type of infertility, endometrial thickness at embryo transfer day, and the duration of treatment, have not influence on the pregnancy rate after FET.

For patients older than 35 years old, the clinical characteristics, dose of gonadotropin, retrieved of oocyte, and number of good embryo transferred were different significantly between the fresh embryo transfers and frozen-thawed blastocyst transfers clinical pregnancy $(57.1 \%$ versus $12.5 \%)$ is also significantly high in fresh embryo transfer than that of frozen-thawed blastocyst (Table 4). Tiitinen et al, Saldeen and Sundstrom, studies revealed through several fresh embryo transfers cycles, this group reached an acceptable cumulative pregnancy rate of $32.7 \%$. concluded that it seems to be beneficial to women who are at increased risk of complications related to multiple pregnancy. ${ }^{9,10}$ High pregnancy rate observed in women with good quality embryos, even in this age group, suggests that embryo morphology, rather than calendar age, determines the chance of pregnancy. Therefore, selection should be on the basis of embryo quality rather than age.

Recently, studies indicated there were high ectopic pregnancy rates in fresh embryo transfer cycles. The reason may be the high gonadotropin in ovarian stimulation cycles. ${ }^{11}$ The statistics in our study showed no difference in the ectopic pregnancy between fresh embryo transfers and frozen-thawed embryo transfers. Maybe, fresh and frozen-thawed embryo transfers were divided into cleavage-stage and blastocyst to analyse. The abortion rate in frozen-thawed embryo transfer was higher than fresh embryo transfer. Some reports supported the view.

Cleavage-stage embryo and blastocyst may inevitably be damaged during cryopreservation. This may damage the ability of development, thus result in abortion. ${ }^{12}$ Aytoz et al indicated the cryopreservation process had no negative impact on the outcome of pregnancy over 20 weeks of gestation. ${ }^{12}$ There were no differences in low-birthweight, birth defects and perinatal death between the fresh embryo transfers and frozen-thawed embryo transfers. ${ }^{13,14}$

\section{CONCLUSION}

The optimal embryo transfer protocol is fresh cleavagestage embryo transfer if the patients are in good conditions. The clinical pregnancy rates in fresh embryo transfer is high than that of frozen-thawed blastocyst.

Funding: No funding sources

Conflict of interest: None declared

Ethical approval: The study was approved by the Institutional Ethics Committee

\section{REFERENCES}

1. Pattinson HA, Hignett M, Dunphy BC, Fleetham JA. Outcome of thaw embryo transfer after cryopreservation of all embryos in patients at risk of ovarian hyperstimulation syndrome. Fertil Steril. 1994;62:1192-6.

2. Check JH, Choe JK, Nazari A, Fox F, Swenson K. Fresh embryo transfer is more effective than frozen for donor oocyte recipients but not for donors. Hum Reprod. 2001;16:1403-8.

3. Shen C, Shu D, Zhao X, Gao Y. Comparison of clinical outcomes between fresh embryo transfers and frozen-thawed embryo transfers. Iran J Reprod Med. 2014;12(6):409-14.

4. Mesut N, Ciray HN, Mesut A, Aksoy T, Bahceci M. Cryopreservation of blastocysts is the most feasible strategy in good responder patients. Fertil Steril. 2011;96:1121-5.

5. Aflatoonian A, Oskouian H, Ahmadi S, Oskouian L. Can fresh embryo transfers be replaced by cryopreserved-thawed embryo transfers in assisted reproductive cycles? A randomized controlled trial. J Assist Reprod Genet. 2010;27:357-63.

6. Ashrafi M, Jahangiri N, Hassani F, Akhoond MR, Madani T. The factors affecting the outcome of frozen-thawed embryo transfer cycle. Taiwan J Obstet Gynecol. 2011;50:159-64.

7. Check JH, Katsoff B, Brasile D, Choe JK, Amui J. Pregnancy outcome following in vitro fertilizationembryo transfer (IVF-ET) in women of more advanced reproductive age with elevated serum follicle stimulating hormone (FSH) levels. Clin Exp Obstet Gynecol. 2008;35:13-5.

8. Kassab A, Sabatini L, Tozer A, Zosmer A, Mostafa $\mathrm{M}$, Al-Shawaf $\mathrm{T}$. The correlation between basal 
serum follicle-stimulating hormone levels before embryo cryopreservation and the clinical outcome of frozen embryo transfers. Fertil Steril. 2009;92:126975 .

9. Tiitinen A, Unkila-Kallio L, Halttunen M, HydènGranskog C. Impact of elective single embryo transfer on the twin pregnancy rate. Hum Reprod. 2003;18:1449-53.

10. Saldeen P, Sundstrom P. Would legislation imposing single embryo transfer be a feasible way to reduce the rate of multiple pregnancies after IVF treatment? Hum Reprod. 2005;20:4-8.

11. Shapiro BS, Daneshmand ST, De Leon L, Garner FC, Aguirre M, Hudson C. Frozen-thawed embryo transfer is associated with a significantly reduced incidence of ectopic pregnancy. Fertil Steril. 2012;98:1490-4.

12. Aytoz A, Van den Abbeel E, Bonduelle M, Camus M, Joris H, Van Steirteghem A, et al. Obstetric outcome of pregnancies after the transfer of cryopreserved and fresh embryos obtained by conventional in-vitro fertilization and intracytoplasmic sperm injection. Hum Reprod. 1999;14:2619-24.

13. Aflatoonian A, Mansoori Moghaddam F, Mashayekhy M, Mohamadian F. Comparison of early pregnancy and neonatal outcomes after frozen and fresh embryo transfer in ART cycles. J Assist Reprod Genet. 2010;27:695-700.

14. Shih W, Rushford DD, Bourne H, Garrett C, McBain JC, Healy DL, et al. Factors affecting low birthweight after assisted reproduction technology: difference between transfer of fresh and cryopreserved embryos suggests an adverse effect of oocyte collection. Hum Reprod. 2008;23:1644-53.

Cite this article as: Anitha A, Rani BS. Comparison of fresh and frozen thawed embryo transfer in terms of clinical pregnancy rate. Int J Reprod Contracept Obstet Gynecol 2020;9:607-12. 